SLAC-PUB-12302

January 2007

\title{
Charm Dalitz Analyses at BABAR
}

by Margo Pappagallo for the BaBar Collaboration

Submitted to Int.J.Mod.Phys. 


\title{
Charm Dalitz analyses at BABAR
}

\author{
MARCO PAPPAGALLO* \\ Representing the BABAR collaboration \\ Institute for Particle Physics Phenomenology, \\ Durham University, South Road, Durham, DH1 3LE, United Kingdom \\ marco.pappagallo@durham.ac.uk
}

Received Day Month Year

Revised Day Month Year

\begin{abstract}
Dalitz plot analyses of $D^{0}$ events reconstructed for the hadronic decay $D^{0} \rightarrow \bar{K}^{0} K^{+} K^{-}$ and $D^{0} \rightarrow \bar{K}^{0} \pi^{+} \pi^{-}$are presented here. The analyses are based on a data sample of $91.5 \mathrm{fb}^{-1}$. All data have been collected with the BABAR detector at the PEP-II asymmetric-energy $e^{+} e^{-}$storage rings at SLAC running at center-of-mass energies on and $40 \mathrm{MeV}$ below the $\Upsilon(4 S)$ resonance.
\end{abstract}

Keywords: Keyword1; keyword2; keyword3.

PACS numbers: $11.25 . \mathrm{Hf}, 123.1 \mathrm{~K}$

\section{Introduction}

Although the BABAR project is mainly known as a $\mathrm{B}$ meson factory there is much more than B physics which can be done at this facility. The copious production of $c \bar{c}$ pairs from the continuum and high integrated luminosity, makes $B A B A R$ an excellent laboratory to study the charm production and decays.

In this paper we show the results on a study of $D^{0} \rightarrow \bar{K}^{0} K^{+} K^{-}$decay $^{1}$ and a measurement of $a_{0}(980)$ meson parameters. A Dalitz plot analysis of $D^{0} \rightarrow \bar{K}^{0} \pi^{+} \pi^{-}$ decay is also shown ${ }^{2,3}$. The latter decay plays a fundamental role in the measurement of the $\gamma$ angle of the Unitarity Triangle. For both decays, the $\bar{K}^{0}$ is detected via the decay $K_{S}^{0} \rightarrow \pi^{+} \pi^{-}$, while the decay $D^{*+} \rightarrow D^{0} \pi^{+}$is used to identify the flavor of the $D^{0}$ (through the charge of the slow $\pi^{ \pm}$from $D^{*}$ decay) and to reduce background. All references to an explicit decay mode, unless otherwise specified, imply the use of the charge conjugate decay as well.

*Affiliated to the BaBar collaboration through University of Warwick, Coventry, CV4 7AL, United Kingdom. 

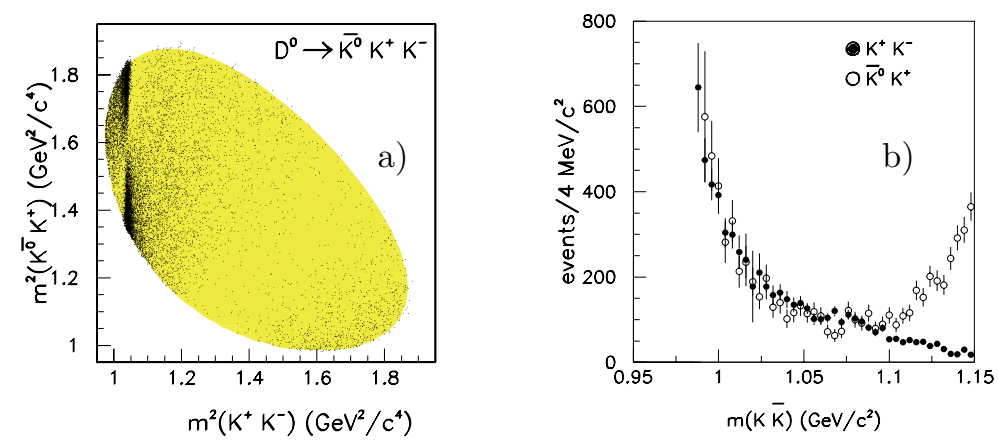

Fig. 1. (a) Dalitz plot of $D^{0} \rightarrow \bar{K}^{0} K^{+} K^{-}$. (b) Comparison between the scalar $K^{+} K^{-}$and the $\bar{K}^{0} K^{+}$phase space corrected mass distributions.

\section{2. $D^{0} \rightarrow \bar{K}^{0} K^{+} K^{-}$decay and scalar mesons}

Charm Dalitz plot analyses are useful in providing new information on resonances that contribute to three-body final states. They can help to enlighten old puzzles related to light meson spectroscopy, specifically to the structure of scalar mesons. The study of $D^{0} \rightarrow \bar{K}^{0} K^{+} K^{-}$decay provides a laboratory to investigate scalar mesons coupling to the $K \bar{K}$ system, in particular the $f_{0}(980)$ and $a_{0}(980)$.

\subsection{Partial wave analysis of $D^{0} \rightarrow \bar{K}^{0} K^{+} K^{-}$}

The $D^{0} \rightarrow \bar{K}^{0} K^{+} K^{-}$sample consists of 12540 events with a signal fraction of $97.3 \%$. The Dalitz plot of the $D^{0} \rightarrow \bar{K}^{0} K^{+} K^{-}$is shown in Fig. 1a. A strong interference between the $\phi(1020)$ and a scalar meson, which is identified as mostly due to the $a_{0}(980)$ resonance, is observed in the low mass $K \bar{K}$ region. The contribution of $a_{0}(980)^{+}$, in the right corner at the bottom, can also be observed. A partial wave analysis in the low mass $K^{+} K^{-}$region allows the $K^{+} K^{-}$scalar $(S)$ and vector components $(P)$ to be separated, thus solving the following system of equations ${ }^{4}$ :

$$
\begin{gathered}
\sqrt{4 \pi}\left\langle Y_{0}^{0}\right\rangle=S^{2}+P^{2} \\
\sqrt{4 \pi}\left\langle Y_{1}^{0}\right\rangle=2|S||P| \cos \phi_{S P} \\
\sqrt{4 \pi}\left\langle Y_{2}^{0}\right\rangle=\frac{2}{\sqrt{5}} P^{2}
\end{gathered}
$$

where $\left\langle Y_{L}^{0}\right\rangle_{L=0,1,2}$ are the efficiency corrected spherical harmonic moments. The resulting scalar $K^{+} K^{-}$and $\bar{K}^{0} K^{+}$mass distributions, corrected for phase space, are displayed in Fig. 1b and show a good agreement. This supports the hypothesis that the $f_{0}(980)$ contribution is small, since $f_{0}(980)$ has isospin zero and therefore cannot decay to $\bar{K}^{0} K^{+}$. 

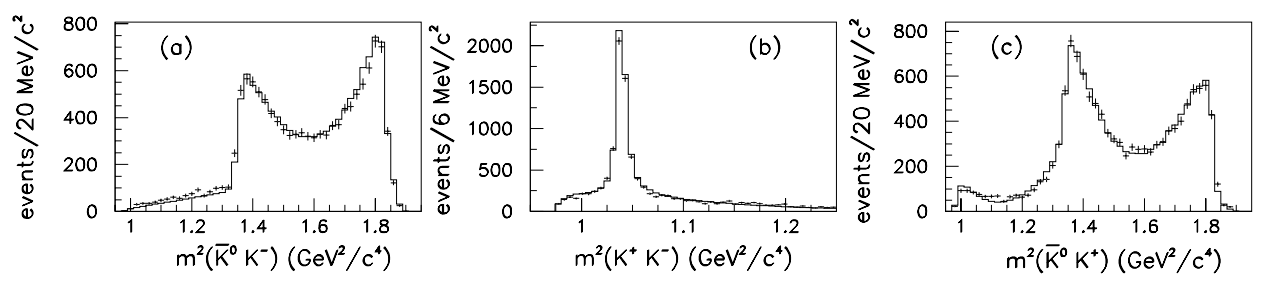

Fig. 2. Dalitz plot projections for $D^{0} \rightarrow \bar{K}^{0} K^{+} K^{-}$. The data are represented with error bars; the histogram is the projection of the fit described in the text.

The $K^{+} K^{-} S$ and $P$ wave mass spectra, the $\bar{K}^{0} K^{+}$mass spectrum and the phase difference $\phi_{S P}$ have been fitted simultaneously where the $K^{+} K^{-} \mathrm{P}$-wave is supposed to be entirely due to the $\phi(1020)$ meson, the $K^{+} K^{-}$S-wave to the $a_{0}(980)^{0}$ and the $\bar{K}^{0} K^{+}$mass distribution to $a_{0}(980)^{+}$. The $a_{0}(980)$ scalar resonance has a mass very close to the $\bar{K} K$ threshold and mostly decays to $\eta \pi$. It has been described by a coupled channel Breit Wigner of the form:

$$
B W_{c h}\left(a_{0}\right)(m)=\frac{g_{\bar{K} K}}{m_{0}^{2}-m^{2}-i\left(\rho_{\eta \pi} g_{\eta \pi}^{2}+\rho_{\bar{K} K} g_{\bar{K} K}^{2}\right)}
$$

where $\rho(m)=2 q / m$ while $g_{\eta \pi}$ and $g_{\bar{K} K}$ describe the $a_{0}(980)$ couplings to the $\eta \pi$ and $\bar{K} K$ systems respectively. Since in the current analysis the only available projections are the $K \bar{K}$ ones, it is not possible to measure $m_{0}$ and $g_{\eta \pi}$. Therefore, these two quantities have been fixed to the Crystal Barrel measurements ${ }^{5}$. The parameter $g_{\bar{K} K}$, on the other hand, has been left free in the fit. The result is (statistical error only):

$$
g_{\bar{K} K}=464 \pm 29(\mathrm{MeV})^{1 / 2} .
$$

\subsection{Dalitz plot analysis of $D^{0} \rightarrow \bar{K}^{0} K^{+} K^{-}$}

An unbinned maximum likelihood fit has been performed for the decay $D^{0} \rightarrow \bar{K}^{0} K^{+} K^{-}$in order to use the distribution of events in the Dalitz plot to determine the relative amplitudes and phases of intermediate resonant and nonresonant states. The $D^{0} \rightarrow \bar{K}^{0} K^{+} K^{-}$decay amplitude $\mathcal{A}_{D}\left(m_{-}^{2}, m_{+}^{2}\right)$ is expressed as a sum of two-body decay-matrix elements and a non-resonant contribution,

$$
\mathcal{A}_{D}\left(m_{-}^{2}, m_{+}^{2}\right)=\Sigma_{r} a_{r} e^{i \phi_{r}} \mathcal{A}_{r}\left(m_{-}^{2}, m_{+}^{2}\right)+a_{\mathrm{NR}} e^{i \phi_{\mathrm{NR}}},
$$

where each term is parametrized with an amplitude $a_{r}$ and a phase $\phi_{r}$. The function $\mathcal{A}_{r}\left(m_{-}^{2}, m_{+}^{2}\right)$ is the Lorentz-invariant expression for the matrix element of a $D^{0}$ meson decaying into $\bar{K}^{0} K^{-} K^{+}$through an intermediate resonance $r$, parametrized as a function of position in the Dalitz plane. We refer to this model as the BreitWigner (or Isobar) model ${ }^{6,7}$. The $D^{0} \rightarrow \bar{K}^{0} K^{+} K^{-}$Dalitz plot projections together with the fit results are shown in Fig. 2. 
The results of the Dalitz plot analysis can be summarized as follows (Tab. 1):

(i) The decay is dominated by $D^{0} \rightarrow \bar{K}^{0} a_{0}(980)^{0}, D^{0} \rightarrow \bar{K}^{0} \phi(1020)$ and $D^{0} \rightarrow K^{-} a_{0}(980)^{+}$

(ii) The $f_{0}(980)$ contribution is consistent with zero;

(iii) The doubly Cabibbo suppressed(DCS) contribution is consistent with zero;

(iv) The remaining contribution, not consistent with being uniform, can be described by the tail of a broad resonance, the $f_{0}(1400)$.

Table 1. Results from the Dalitz plot analysis of $D^{0} \rightarrow \bar{K}^{0} K^{+} K^{-}$. The fits have been performed using the value of $g_{\bar{K} K}$ resulting from the partial wave analysis.

\begin{tabular}{lccc}
\hline Final state & Amplitude & Phase(radians) & Fraction(\%) \\
\hline $\bar{K}^{0} a_{0}(980)^{0}$ & 1.(fixed) & 0.(fixed) & $66.4 \pm 1.6 \pm 7.0$ \\
$\bar{K}^{0} \phi(1020)$ & $0.437 \pm 0.006 \pm 0.060$ & $1.91 \pm 0.02 \pm 0.10$ & $45.9 \pm 0.7 \pm 0.7$ \\
$K^{-} a_{0}(980)^{+}$ & $0.460 \pm 0.017 \pm 0.056$ & $3.59 \pm 0.05 \pm 0.20$ & $13.4 \pm 1.1 \pm 3.7$ \\
$\bar{K}^{0} f_{0}(1400)$ & $0.435 \pm 0.033 \pm 0.162$ & $-2.63 \pm 0.10 \pm 0.71$ & $3.8 \pm 0.7 \pm 2.3$ \\
$\bar{K}^{0} f_{0}(980)$ & & & $0.4 \pm 0.2 \pm 0.8$ \\
$K^{+} a_{0}(980)^{-}$DCS & & & $0.8 \pm 0.3 \pm 0.8$ \\
\hline Sum & & & $130.7 \pm 2.2$ \\
\hline
\end{tabular}

\section{CKM $\gamma$ angle and the $D^{0} \rightarrow K_{S}^{0} \pi^{+} \pi^{-}$decay}

Various methods ${ }^{8-11}$ have been proposed to extract the angle $\gamma$ of the Unitarity Triangle using $B^{-} \rightarrow \tilde{D}^{0} K^{-}$decays, all exploiting the interference between the color allowed $B^{-} \rightarrow D^{0} K^{-}\left(\propto V_{c b}\right)$ and the color suppressed $B^{-} \rightarrow \bar{D}^{0} K^{-}\left(\propto V_{u b}\right)$ transitions, when the $D^{0}$ and $\bar{D}^{0}$ are reconstructed in a common final state. The symbol $\tilde{D}^{0}$ indicates either a $D^{0}$ or a $\bar{D}^{0}$ meson. Among the $\tilde{D}^{0}$ decay modes studied so far the $K_{S}^{0} \pi^{-} \pi^{+}$channel is the one with the highest sensitivity to $\gamma$ because of the best overall combination of branching ratio magnitude, $D^{0}-\bar{D}^{0}$ interference and background level.

\subsection{Dalitz plot analysis of $D^{0} \rightarrow K_{S}^{0} \pi^{+} \pi^{-}$}

The $D^{0} \rightarrow K_{S}^{0} \pi^{+} \pi^{-}$sample consists of 81496 events with a signal fraction of $97 \%$. The Dalitz plot of the $D^{0} \rightarrow K_{S}^{0} \pi^{+} \pi^{-}$is shown in Fig. 3a.

Table 2 summarizes the values of $a_{r}$ and $\phi_{r}$ obtained using a Breit-Wigner model consisting of 16 two-body elements comprising doubly Cabibbo suppressed contribution, and accounting for efficiency variations across the Dalitz plane and the small background contribution. We find that the inclusion of the scalar $\pi \pi$ resonances $\sigma$ and $\sigma^{\prime}$ significantly improves the quality of the fit ${ }^{12}$. Since the two $\sigma$ resonances are not well established and are only introduced to improve the description of our data, the uncertainty depending on them is included in the systematic errors. Goodness of fit is gauged through a two-dimensional $\chi^{2}$ test, thus obtaining $\chi^{2}=1.27$. 

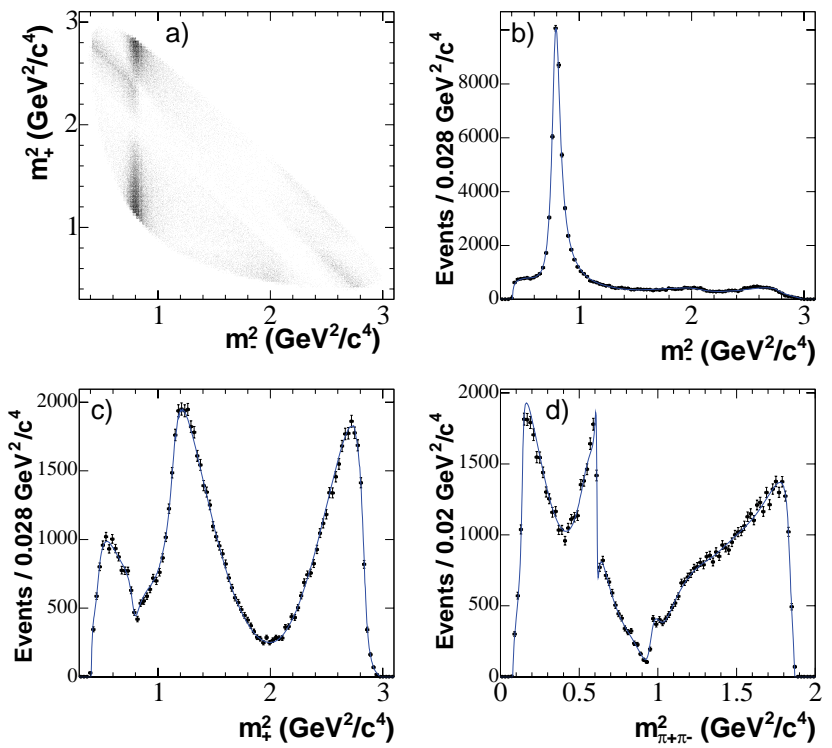

Fig. 3. (a) The $D^{0} \rightarrow K_{S}^{0} \pi^{-} \pi^{+}$Dalitz distribution. $m_{-}^{2}$ and $m_{+}^{2}$ are the squared invariant masses of the $K_{S}^{0} \pi^{-}$and $K_{S}^{0} \pi^{+}$combinations respectively. (b) Dalitz plot projections on $m_{-}^{2}$, (c) $m_{+}^{2}$, and (d) $m_{\pi^{+} \pi^{-}}^{2}$. The curves are the fit projections.

Table 2. Amplitudes $a_{r}$, phases $\phi_{r}$ and fit fractions obtained from the fit of the $D^{0} \rightarrow K_{S}^{0} \pi^{+} \pi^{-}$Dalitz distribution. Errors are statistical only. The sum of fit fractions is 1.24 .

\begin{tabular}{lccc}
\hline Component & Amplitude & Phase $(\mathrm{deg})$ & Fit fraction \\
\hline$K^{*}(892)^{-}$ & $1.781 \pm 0.018$ & $131.0 \pm 0.8$ & 0.586 \\
$K_{0}^{*}(1430)^{-}$ & $2.45 \pm 0.08$ & $-8.3 \pm 2.5$ & 0.083 \\
$K_{2}^{*}(1430)^{-}$ & $1.05 \pm 0.06$ & $-54.3 \pm 2.6$ & 0.027 \\
$K^{*}(1410)^{-}$ & $0.52 \pm 0.09$ & $154 \pm 20$ & 0.004 \\
$K^{*}(1680)^{-}$ & $0.89 \pm 0.30$ & $-139 \pm 14$ & 0.003 \\
\hline$K^{*}(892)^{+}$DCS & $0.180 \pm 0.008$ & $-44.1 \pm 2.5$ & 0.006 \\
$K_{0}^{*}(1430)^{+}$DCS & $0.37 \pm 0.07$ & $18 \pm 9$ & 0.002 \\
$K_{2}^{*}(1430)^{+}$DCS & $0.075 \pm 0.038$ & $-104 \pm 23$ & 0.000 \\
\hline$\rho(770)$ & 1 (fixed) & 0 (fixed) & 0.224 \\
$\omega(782)$ & $0.0391 \pm 0.0016$ & $115.3 \pm 2.5$ & 0.006 \\
$f_{0}(980)$ & $0.482 \pm 0.012$ & $-141.8 \pm 2.2$ & 0.061 \\
$f_{0}(1370)$ & $2.25 \pm 0.30$ & $113.2 \pm 3.7$ & 0.032 \\
$f_{2}(1270)$ & $0.922 \pm 0.041$ & $-21.3 \pm 3.1$ & 0.030 \\
$\rho(1450)$ & $0.52 \pm 0.09$ & $38 \pm 13$ & 0.002 \\
$\sigma$ & $1.36 \pm 0.05$ & $-177.9 \pm 2.7$ & 0.093 \\
$\sigma^{\prime}$ & $0.340 \pm 0.026$ & $153.0 \pm 3.8$ & 0.013 \\
\hline Non Resonant & $3.53 \pm 0.44$ & $128 \pm 6$ & 0.073 \\
\hline
\end{tabular}

\subsection{Dalitz plot analysis of $D^{0} \rightarrow K_{S}^{0} \pi^{+} \pi^{-}$with $\pi \pi S$-wave parametrized by a K-matrix model}

In order to investigate the effect of the chosen model on the angle $\gamma$, a model using the K-matrix formalism ${ }^{13-15}$ to parametrize the $S$-wave component of the $\pi \pi$ 
system in $D^{0} \rightarrow K_{S}^{0} \pi^{-} \pi^{+}$is used. The K-matrix approach provides a direct way of imposing the unitarity constraint that is not guaranteed in the case of the BreitWigner model. Therefore, the K-matrix method is suited to the study of broad and overlapping resonances in multi-channel decays, solving the main limitation of the Breit-Wigner model to parametrize the $\pi \pi S$-wave states in $D^{0} \rightarrow K_{S}^{0} \pi^{-} \pi^{+16}$.

The Dalitz amplitude $\mathcal{A}_{D}\left(m_{-}^{2}, m_{+}^{2}\right)$ is written in such a case as a sum of twobody decay matrix elements for the spin- 1 , spin- 2 and $K \pi$ spin- 0 resonances (as in the Breit-Wigner model), and the $\pi \pi$ spin- 0 piece denoted as $F_{1}$ is written in terms of the K-matrix. Therefore we have:

$$
\mathcal{A}_{D}\left(m_{-}^{2}, m_{+}^{2}\right)=F_{1}(s)+\Sigma_{r \neq \pi \pi \mathrm{S}-\text { wave }} a_{r} e^{i \phi_{r}} \mathcal{A}_{r}\left(m_{-}^{2}, m_{+}^{2}\right)
$$

Table 3 summarizes the values of $F_{1}(s)$ free parameters $\beta_{\alpha}$ and $f_{11}^{\text {prod }}$, together with the spin-1, spin-2, and $K \pi$ spin- 0 amplitudes as in the Breit-Wigner model. Figures $3(\mathrm{~b}, \mathrm{c}, \mathrm{d})$ show the fit projections overlaid with the data distributions. There is no overall improvement in the two-dimensional $\chi^{2}$ test compared to the BreitWigner model since it is dominated by the $\mathrm{P}$-wave components, which are identical in both models. Nevertheless, it should be emphasized that the main advantage in using a K-matrix parametrization instead of a sum of two-body amplitudes to describe the $\pi \pi \mathrm{S}$-wave is that it provides a more adequate description of the complex dynamics in the presence of overlapping and many channel resonances.

Table 3. Complex amplitudes $a_{r} e^{i \phi_{r}}$ and fit fractions of the different components obtained from the fit of the $D^{0} \rightarrow K_{S}^{0} \pi^{-} \pi^{+}$Dalitz distribution. Errors are statistical only. The sum of fit fractions is 1.16 .

\begin{tabular}{l|ccc}
\hline Component & $\operatorname{Re}\left\{a_{r} e^{i \phi_{r}}\right\}$ & $\operatorname{Im}\left\{a_{r} e^{i \phi_{r}}\right\}$ & Fit fraction (\%) \\
\hline \hline$K^{*}(892)^{-}$ & $-1.159 \pm 0.022$ & $1.361 \pm 0.020$ & 58.9 \\
$K_{0}^{*}(1430)^{-}$ & $2.482 \pm 0.075$ & $-0.653 \pm 0.073$ & 9.1 \\
$K_{2}^{*}(1430)^{-}$ & $0.852 \pm 0.042$ & $-0.729 \pm 0.051$ & 3.1 \\
$K^{*}(1410)^{-}$ & $-0.402 \pm 0.076$ & $0.050 \pm 0.072$ & 0.2 \\
$K^{*}(1680)^{-}$ & $-1.00 \pm 0.29$ & $1.69 \pm 0.28$ & 1.4 \\
\hline$K^{*}(892)^{+}$DCS & $0.133 \pm 0.008$ & $-0.132 \pm 0.007$ & 0.7 \\
$K_{0}^{*}(1430)^{+}$DCS & $0.375 \pm 0.060$ & $-0.143 \pm 0.066$ & 0.2 \\
$K_{2}^{*}(1430)^{+}$DCS & $0.088 \pm 0.037$ & $-0.057 \pm 0.038$ & 0.0 \\
\hline$\rho(770)$ & $1($ fixed $)$ & $0($ fixed $)$ & 22.3 \\
$\omega(782)$ & $-0.0182 \pm 0.0019$ & $0.0367 \pm 0.0014$ & 0.6 \\
$f_{2}(1270)$ & $0.787 \pm 0.039$ & $-0.397 \pm 0.049$ & 2.7 \\
$\rho(1450)$ & $0.405 \pm 0.079$ & $-0.458 \pm 0.116$ & 0.3 \\
\hline \hline$\beta_{1}$ & $-3.78 \pm 0.13$ & $1.23 \pm 0.16$ & - \\
$\beta_{2}$ & $9.55 \pm 0.20$ & $3.43 \pm 0.40$ & - \\
$\beta_{4}$ & $12.97 \pm 0.67$ & $1.27 \pm 0.66$ & - \\
$f_{11}^{\text {prod }}$ & $-10.22 \pm 0.32$ & $-6.35 \pm 0.39$ & - \\
sum of $\pi^{+} \pi^{-} S$-wave & & & 16.2 \\
\hline
\end{tabular}

From $B^{\mp} \rightarrow D^{(*)} K^{\mp}$ and $B^{\mp} \rightarrow D K^{* \mp}$ decays ${ }^{3}$, we measure $\gamma=(67 \pm 28 \pm 13 \pm 11)^{\circ}$, where the first error is statistical, the second one is the experimental systematic uncertainty and the third one reflects the Dalitz model 
uncertainty. The contribution to the Dalitz model uncertainty due to the description of the $\pi \pi$ S-wave in $D^{0} \rightarrow K_{S}^{0} \pi^{-} \pi^{+}$, evaluated using a K-matrix formalism, is found to be $3^{\circ}$.

\section{Acknowledgments}

The author is grateful for the extraordinary contributions of our PEP-II colleagues in achieving the excellent luminosity and machine conditions that have made this work possible. The collaborating institutions wish to thank SLAC for its support and kind hospitality. This work is supported by DOE and NSF (USA), NSERC (Canada), IHEP (China), CEA and CNRS-IN2P3 (France), BMBF and DFG (Germany), INFN (Italy), FOM (The Netherlands), NFR (Norway), MIST (Russia), and PPARC (United Kingdom). Individuals have received support from CONA-

CyT (Mexico), the A. P. Sloan Foundation, the Research Corporation, and the Alexander von Humboldt Foundation.

The author acknowledges the support of the EU-RTN Programme, Contract No. HPRN-CT-2002-00311, "EURIDICE".

\section{References}

1. BABAR Collab. (B. Aubert et al.), Phys. Rev. D 72, 052008 (2005).

2. BABAR Collab. (B. Aubert et al.), Phys. Rev. Lett. 95, 121802 (2005).

3. BABAR Collab. (B. Aubert et al.), hep-ex/0507101.

4. S.U. Chung, Phys.Rev. D 56, 7299 (1997).

5. A. Abele et al., Phys. Rev. D 57, 3860 (1998).

6. CLEO Collab. (S. Kopp et al.), Phys. Rev. D 63, 092001 (2001).

7. CLEO Collab. (H. Muramatsu et al.), Phys. Rev. Lett. 89, 251802 (2002); Erratum-ibid: 90059901 (2003).

8. M. Gronau and D. London, Phys. Lett. B 253, 483 (1991);

9. M. Gronau and D. Wyler, Phys. Lett. B 265, 172 (1991);

10. D. Atwood, I. Dunietz and A. Soni, Phys. Rev. Lett. 78, 3257 (1997).

11. A. Giri, Yu. Grossman, A. Soffer and J. Zupan, Phys. Rev. D 68, 054018 (2003).

12. The $\sigma$ and $\sigma^{\prime}$ masses and widths are determined from the data. We find (in $\mathrm{MeV} / c^{2}$ ) $M_{\sigma}=484 \pm 9, \Gamma_{\sigma}=383 \pm 14, M_{\sigma^{\prime}}=1014 \pm 7$, and $\Gamma_{\sigma^{\prime}}=88 \pm 13$. Errors are statistical.

13. E. P. Wigner, Phys. Rev. 70 (1946) 15;

14. S. U. Chung et al., Ann. Physik 4 (1995) 404.

15. I. J. R. Aitchison, Nucl. Phys. A 189, 417 (1972).

16. Review on Scalar Mesons in Ref. 17.

17. Particle Data Group, S. Eidelman et al., Phys. Lett. B 592, 1 (2004). 